\title{
The management of rectal bleeding following transrectal prostate biopsy: A review of the current literature
}

\author{
Mark R. Quinlan ${ }^{1}$; Damien M. Bolton ${ }^{1}$; Rowan G. Casey ${ }^{2}$ \\ ${ }^{1}$ Department of Urology, Austin Hospital, Heidelberg, Melbourne, Australia; ${ }^{2}$ Department of Urology, \\ Colchester Cancer Centre, Colchester NHS University Foundation Trust, Essex, United Kingdom
}

Cite as: Can Urol Assoc J 2017 Dec. 22; Epub ahead of print.

http://dx.doi.org/10.5489/cuaj.4660

Published online December 22, 2017

$* * *$

\begin{abstract}
Introduction: Since the advent of prostate-specific antigen (PSA)-based testing, transrectal ultrasound (TRUS)-guided prostate biopsy has become a standard part of the diagnostic pathway for prostate cancer (PCa). Rectal bleeding is one of the common side effects of this transrectal route. While rectal bleeding is usually mild and self-limiting, it can be lifethreatening. In this article, we examine rectal bleeding post TRUS-guided prostate biopsy and explore the literature to evaluate techniques and strategies aimed at preventing and managing this common and important complication.
\end{abstract}

Methods: A PubMed literature search was carried out using the keywords "transrectalprostate-biopsy-bleed." A search of the bibliography of reviewed studies was also conducted. Additionally, papers in non-PubMed-listed journals of which the authors were aware were appraised.

Results: Numerous modifiable risk factors for this bleeding complication exist, particularly anticoagulants/antiplatelets and the number of core biopsies taken. Successfully described corrective measures for such rectal bleeding include tamponade (digital/packs/catheter/tampon/condom), endoscopic sclerotherapy/banding/clipping, radiological embolization, and surgical intervention.

Conclusions: We advocate early consultation with the colorectal/gastroenterology and interventional radiology services and a progressive, stepwise approach to the management of post-biopsy rectal bleeding, starting with resuscitation and conservative tamponade measures, moving to endoscopic hemostasis \pm radiological embolization \pm transanal surgical methods. Given the infrequent but serious nature of major rectal bleeding after TRUS biopsy, we recommend the establishment of centralized databases or registries forthwith to prospectively capture such data. To the best of our knowledge, this is the first comprehensive look specifically at the management of post-TRUS biopsy rectal bleeding. 


\section{Introduction}

Central to PSA-based testing for PCa is the role of TRUS-guided prostate biopsy. It is estimated that more than one million TRUS-guided prostate biopsies are currently performed annually in both the USA and Europe. ${ }^{1}$ Furthermore, active surveillance (AS) protocols for low-risk PCa have become established in various guidelines, including the European Association of Urology (EAU) PCa guidelines. ${ }^{2}$ AS typically involves repeat prostate biopsies at predefined follow-up intervals, often as frequently as yearly. ${ }^{3,4}$ Thus, as AS gains increasing acceptance, the number of prostate biopsies will increase. ${ }^{5}$

TRUS-guided prostate biopsy can cause several potential complications, including haematospermia (37.4\%), haematuria (14.5\%), rectal bleeding $<2$ days $(2.2 \%)$, rectal bleeding $>2$ days $+/$ - intervention $(0.7 \%)$, sepsis $(0.8 \%)$ or urinary retention $(0.2 \%) .{ }^{2}$ The literature reports higher rectal bleeding rates - in one prospective study, $25 \%$ of patients experienced a rectal bleed after biopsy. ${ }^{6}$ Enlund et al. also showed a $22 \%$ immediate post biopsy rate of haematochezia which fell to $3 \%$ at three days and $0.5 \%$ at seven days. ${ }^{7}$ Massive rectal haemorrhage post-biopsy has been reported in up to $1 \%$ of cases. ${ }^{8} \mathrm{Al}$-Otaibi et al. report a case of rectal bleeding precipitating disseminated intravascular coagulation which required intensive care unit (ICU) treatment and correction of acute renal failure, pulmonary oedema and atrial fibrillation. ${ }^{9}$ A large anterior rectal wall haematoma with near total occlusion of the rectal lumen following a TRUS-guided prostate biopsy has also been reported and sizeable prevesical space of Retzius haematomas have likewise been described. ${ }^{10,11}$ Furthermore, case reports describe severe rectal bleeding more than two weeks after prostate biopsy requiring endoscopic haemostatic treatment. ${ }^{12}$ Severe rectal bleeding is thus an uncommon but potentially life-threatening complication and one of which both patients and practitioners should be aware. ${ }^{13}$ Post biopsy rectal bleeding is most commonly just a Clavien-Dindo Grade I complication but as outlined above, on occasion, it can result in grade II (eg: blood transfusion required)-IV (organ dysfunction and ICU management) sequelae.

Transperineal (TP) and MRI-fusion biopsies are emerging as alternatives to TRUS biopsy but they have not yet replaced TRUS biopsy due to their increased costs, requirement for general anaesthesia (GA) and lack of widespread availability. In the case of TP biopsies, in one study, a haemorrhagic complication of any type occurred in just $1.8 \%$ of cases and none were reported as severe. ${ }^{14}$ A comparative study of TRUS biopsy versus TP biopsy showed remarkably greater incidence rates of post-biopsy rectal bleeding (50.5\% vs $3.4 \%$, P $>0.01$ ) in the former group. ${ }^{15}$ MRI-guided prostate biopsy appears to offer no advantage over traditional TRUS biopsy in terms of incidence and duration of bleeding complications. ${ }^{16}$ The prostate and surrounding rectal tissue are supplied by a rich vascular network consisting of branches of the Inferior Vesical Artery and the Superior (SRA), Middle (MRA) and Inferior Rectal (IRA) arteries. The rectal venous plexus is also dense in the submucosal space of this region. Specifically, reports suggest that it may be the SRA and MRA that bleed after biopsy. ${ }^{13,17}$ Other reports suggest it is the rectal venous plexus which bleeds. ${ }^{18}$ Regardless of the precise bleeding source, the anterior rectal wall is classically the location of the 
haemorrhage. ${ }^{8,19}$ Baum et al. contend that rectal bleeding occurs every 15 minutes as the ampulla fills with blood stimulating the urge to evacuate. ${ }^{20}$

This review examines rectal bleeding post TRUS-guided prostate biopsy. We explore the literature to evaluate risk factors (RFs) for its development and techniques aimed primarily at managing but also preventing this infrequent but occasionally very significant complication.

\section{Methods}

A Pubmed literature search was conducted using the keywords "transrectal-prostate-biopsybleed”. This search yielded 144 "hits” going back to 1970.62 of these were immediately dismissed as they weren't directly relevant to our review topic. Not all of the 82 papers that were reviewed were ultimately included in our bibliography as some merely echoed what other papers had reported while others added little. A manual search of the bibliography of reviewed studies was also conducted. Additional papers in non-Pubmed listed journals of which the authors were aware were also appraised. Published guidelines from the EAU were included in the literature search process. We also included an "epidemiological” paper which looked at a classification system for surgical techniques. In all, 135 papers were reviewed. 68 are cited in our bibliography- see Figure 1. Eight papers provide level I evidence and two provide level IV evidence. All others provide level II or III evidence - see Table 1. Importantly, there is no consistent or specific definition for what constitutes "severe" or "massive" or "life-threatening" bleeding but haemodynamic instability and the need for blood transfusion were taken as evidence of such bleeding. To the best of our knowledge, this is the first comprehensive review paper looking specifically at post-TRUS biopsy rectal bleeding.

\section{Results}

\section{Risk factors (RFs)}

Several modifiable RFs which may increase the likelihood of post-TRUS biopsy rectal bleeding have been described.

\section{Anticoagulants/antiplatelets (see Table 2)}

Much attention has understandably focused on the widespread use of anticoagulant and antiplatelet agents, with Aspirin being the most ubiquitous of these and thus the most comprehensively studied. Definitive guidelines regarding the management of these medications before a TRUS biopsy are yet to be established. One study reports that nearly $60 \%$ of consultant members of the British Association of Urological Surgeons will continue Aspirin prior to a TRUS-guided prostate biopsy while only $8 \%$ will continue Clopidogrel and $5 \%$ will continue Warfarin. ${ }^{21}$ Some studies suggest that patients taking Aspirin have a significantly higher cumulative incidence of rectal bleeding, though these are considered only minor bleeding complications. ${ }^{22,23}$ Conversely, others argue that Aspirin does not increase the incidence of rectal bleeding and that Aspirin does not need to be discontinued beforehand. ${ }^{24}$ 
In Carmignani's meta-analysis, the occurrence of rectal bleeding was not statistically increased in patients taking Aspirin and so it was concluded that stopping Aspirin before TRUS biopsy is unnecessary. ${ }^{25}$ Another meta-analysis concluded that low-dose Aspirin neither increases the level of the severity of bleeding complications nor the perioperative mortality because of bleeding complications while discontinuing it increases perioperative cardiovascular risks with life threatening sequelae. ${ }^{26}$ In a review paper by the American Urological Association on anticoagulation and antiplatelet therapy in Urological practice, the authors conclude that uninterrupted use of Aspirin does not increase the risk of rectal bleeding after TRUS biopsy. ${ }^{27}$ Thus, they recommend continuing Aspirin for patients with moderate/high thromboembolic risks.

Less information is available regarding those taking Clopidogrel or Warfarin. In one of the few studies published, the frequency and severity of bleeding complications were no worse in the Warfarin group than in the control group and the authors concluded that its discontinuation before prostate biopsy may again be unnecessary. ${ }^{28}$ Similarly, Halliwell et al. contend that the theoretical risk of a life-threatening bleeding complication in the patient group taking Warfarin is outweighed by the risk of cardio- and cerebro-vascular accidents (CVA) with the cessation of the medication. ${ }^{29}$ The risk of CVA with a subtherapeutic INR is estimated to be $0.003 \%-0.005 \%$ per day. ${ }^{30}$

Raheem et al. looked at anticoagulants and antiplatelets as a combined group and concluded that it is unnecessary to cease these agents before TRUS biopsy. ${ }^{31}$ A total of 91 and 98 patients were included in their anticoagulation/antiplatelet (monotherapy of Aspirin, Warfarin, Clopidogrel, or Low Molecular Weight Heparin or dual therapy of Aspirin and Warfarin or Clopidogrel) and control groups, respectively. The median INR for Warfarin patients was 2.35. Using a 12-core peripheral zone technique, the authors found the incidence of rectal bleeding to be similar in those taking $\leq$ two anticoagulants $(40 \%)$ and those taking none (39\%). Little has yet been reported about the effect of new oral anticoagulants (NOACs) on prostate biopsy bleeding rates although one study does recommend discontinuing such agents five days pre-biopsy, bridging with therapeutic Heparin and then restarting the NOAC 6-8 hours after biopsy in the absence of worrisome bleeding. ${ }^{32}$

Interestingly, Asano et al. report no statistical difference with regard to the incidence of rectal bleeding following TP prostate biopsy between those taking antithrombotic agents and those not. $^{14}$

\section{Antibiotics}

Prophylactic antibiotics are routinely prescribed pre-biopsy. The Quinolones (especially Ciprofloxacin) are most commonly used as they have good pathogen coverage and excellent prostate penetration. Common alternatives are Co-trimoxazole, Cephalosporins, and Metronidazole. All of these antibiotics facilitate the action of Warfarin and this is another important factor which practitioners must consider when prescribing perioperative Warfarin. ${ }^{33}$ 


\section{Core biopsy number}

According to the literature, the incidence of rectal bleeding following TRUS biopsy varies from $1.3 \%-58.6 \%$, with a statistically significant positive correlation to the number of core samples obtained. ${ }^{34}$ Specifically, rectal bleeding is significantly more prevalent in 12-core prostate biopsy groups than six-core groups; 12-core biopsies at a minimum are now considered the gold standard, with six-core biopsies deemed insufficient for whole gland sampling. $^{35,36,37}$

\section{Local anaesthetic}

Debate exists as to the benefit of local anaesthetic (LA) administration with regards to postbiopsy rectal bleeding. Some argue that LA does not reduce the risk whereas other groups contend that LA is associated with a decreased incidence of this complication, presumably due to decreased patient discomfort and less patient movement. ${ }^{35,38}$ The addition of Epinephrine to Xylocaine anaesthetic solution diminished the incidence of rectal bleeding in one study. ${ }^{39}$

\section{Other RFs}

Other RFs for post-biopsy rectal haemorrhage include the presence of haemorrhoids. ${ }^{40}$ The aforementioned rectal venous plexus can increase in size in cases of haemorrhoids. ${ }^{41}$ Some authors advise preliminary proctoscopy if haemorrhoids are suspected. Sheikh et al. recommend a TP rather than a transrectal biopsy in patients with severe haemorrhoids. ${ }^{42}$ Rietbergen et al. reported an increasing trend of rectal bleeding with advancing age. ${ }^{43}$ Poorly controlled hypertension and constipation have also been mooted as potential RFs which should be corrected prior to the procedure. ${ }^{44}$ The same authors propose that neovascularization related to large volume, high grade PCa may also contribute to excessive bleeding. Specifically, hypoechoic areas in the prostate are usually hypervascular - biopsying these areas might be an additional RF for bleeding but the dilemma is that these are the precise areas most in need of sampling. ${ }^{45}$

\section{Management}

We classified the papers in the below management section according to McCulloch's descriptive model for surgical procedures/techniques delineating stages of Innovation, Development, Exploration, Assessment, and Long-term study (the IDEAL model). ${ }^{46}$ To the best of our knowledge, one paper meets the criteria for the Development stage, three papers meet the criteria for the Exploration phase, one paper meets the criteria for the Assessment stage while all others fall into the Innovation phase.

\section{Conservative measures}

As with any form of bleeding, initial management of massive post-TRUS biopsy rectal bleeding starts with resuscitation according to Advanced Life Support protocols and blood transfusion may be necessary. ${ }^{44}$ Rectal bleeding is traditionally managed with tamponade as the first conservative method. Digital compression of rectal bleeding can be successful in controlling bleeding. ${ }^{44}$ Rectal packs may be left in situ for a few hours and removed slowly 
after the bleeding has abated. ${ }^{45}$ Tamponade by means of a transrectally inserted urethral catheter (UC), tampon or condom is also well described in tackling rectal bleeding postbiopsy. ${ }^{40,45,47,48}$ These are left in situ until bleeding stops - this is typically 30-60 minutes in the case of tampons. ${ }^{48}$ The normal rectal tone holds these devices against the anterior rectal wall. One such technique involved a 28Fr Foley UC passed through a rectal sponge and inserted with finger-guidance into the rectum - the UC balloon was inflated with $20 \mathrm{ml}$ of water to provide effective tamponade. ${ }^{49}$ Baum's UC technique involves putting the patient in the supine position, passing a UC per rectum and inflating it with $45 \mathrm{mls}$ of water before connecting it to straight drainage. ${ }^{20}$ Traction is applied for 45 minutes with the UC taped to the inner thigh. The extent of bleeding can be observed by viewing the contents of the drainage bag.

Even bigger, $60 \mathrm{ml}$ balloon three-way UCs have been described. ${ }^{47}$ Still, some authors contest that Foley UC balloons are not large enough to be appropriately placed over the bleeding site. The condom balloon tamponade was first reported by Gonen et al. in 2004 $200 \mathrm{mls}$ of water was used to inflate this for two hours. ${ }^{45}$ This device was made by inserting a UC into a condom, which was fixed to the UC with sterile silk. The condom balloon tamponade is larger than the Foley balloon tamponade and is easily placed over the bleeding site. ${ }^{45}$ Recently, Laracy et al. reported the use of thrombin gel to achieve haemostasis after large volume rectal bleeding. ${ }^{50}$

One method of attempting to prophylactically lower the rate of bleeding involves the insertion of a Foley UC into the rectum with inflation of the balloon to 50cc after biopsy. This has previously been shown to significantly reduce bleeding per rectum from $17.7 \%$ in the control group (UC inserted but balloon not inflated) to $1.5 \%$ in the non-control group. ${ }^{51}$ More recently, the insertion of a gelatin sponge into the rectum after biopsy increased haemostasis without increasing patient symptoms. ${ }^{52}$ Park et al. compared TRUS-guided compression on bleeding biopsy tracts immediately after prostate biopsy versus a noncompression group and found that rectal bleeding incidence was significantly lower in the compression group. ${ }^{53}$

\section{Endoscopic correction}

Failure of a conservative approach usually mandates an endoscopic attempt at haemostasis. Indeed, early consultation with a Gastroenterologist/Colorectal surgeon with colonoscopic experience is strongly advised. Endoscopic haemostatic measures represent a safe and efficient method to control serious rectal bleeding. Sclerotherapy has been used to successfully treat life-threatening rectal bleeding and is well described. ${ }^{54}$ Endoscopic injection of Adrenaline has traditionally been the next management step after unsuccessful conservative measures, with doses of $25 \mathrm{ml}$ of 1:10,000 Adrenaline solution reported. ${ }^{55}$ Another sclerosing agent Polidocanol also has been injected successfully. ${ }^{8}$ In Brullet's case series of 550 consecutive patients, five (1\%) presented with rectal bleeding and hypovolaemic symptoms shortly after TRUS biopsy. ${ }^{8}$ Endoscopic injection of Adrenaline and Polidocanol achieved control of bleeding and permanent haemostasis in all. Similarly, endoscopic haemostasis with $1 \%$ Athexysclerol has been achieved. ${ }^{19}$ 
Endoscopic banding has been deployed successfully. ${ }^{56,57}$ The bleeding point is controlled with artery forceps passed through the ring of the banding device, with the band mounted ready for application. After the band has been fired, the artery forceps are released. The band remains in situ, securing the haemostasis and sloughing off a few days later. ${ }^{57}$ The placement of endoscopic clips is now used in some centres - see Figure $2 .^{41}$ Use of endoclipping has been widely reported in Gastrointestinal endoscopy for many years and so this is a potentially promising option for the management of TRUS-biopsy rectal bleeding. ${ }^{58}$ Endoclips dislodge spontaneously and are passed in the faeces without complication. ${ }^{41}$ Endoscopic thermocoagulation using a 10F multipolar probe with five pulses of two to four seconds each is another method described to stop rectal bleeding. ${ }^{59}$ Similarly, both Arroja and Geraci report successful treatment of a major post-biopsy bleed with argon plasma coagulation. ${ }^{60,61}$

\section{Arterial embolization}

Historically, pelvic urological embolization consisted of Internal Iliac Artery embolization for uncontrollable haemorrhage from prostate and bladder malignancies. ${ }^{62}$ More recently, prostatic arterial embolization for benign prostatic hyperplasia has been tried. ${ }^{63}$ In the emergency setting, angiographic embolization has been performed to address catastrophic bleeding post-TRUS biopsy in patients deemed too unstable for colonoscopy. ${ }^{64,65}$ It is considered to be fast, safe and accurate. The SRA was the source of bleeding in the former paper, believed to be the first reported case of severe life threatening rectal bleeding following TRUS-guided prostate biopsy effectively managed by angiographic therapy. Elsewhere, selective arterial embolization has successfully resulted in haemostasis of a large pelvic haematoma after biopsy. ${ }^{66}$ Additionally, De Beule et al. report a rare case of a postbiopsy prostate arteriovenous fistula resulting in massive rectal haemorrhage which required embolization with calibrated microparticles and microcoils after medical and endoscopic interventions had failed (see Figure 3). ${ }^{67}$ Elsewhere, intra-arterial embolization with Gianturco-Wallace coils was performed on a post-biopsy pulsatile perineal haematoma due to a pseudoaneurysm of the Left Hypogastric Artery. ${ }^{68}$

\section{Surgical manoeuvres}

GA and transrectal surgical correction of bleeding - oversewing of rectal bleeding points +/packing of the rectum with Gelfoam - has been reported as an option. ${ }^{13}$ This may be performed by dilating the anus with a Parke's retractor and suturing bleeding points in the rectal wall with a 3/0 Vicryl suture. In the above case report, this technique was reported as successful when rectal tamponade with a UC, colonoscopy and angiography had all failed to control the bleeding site. However, it's equally important to consider surgical correction as a viable upfront option, depending on resources and circumstances, and not merely a last resort when other techniques have failed. 


\section{Conclusion}

TRUS-guided prostate biopsy has become a common procedure since the advent of PSA testing. Rectal bleeding is acknowledged as one of its common side effects. While this is usually mild and self-limiting, it can potentially be deadly. Debate exists as to the risk presented by anticoagulant/antiplatelet use at the time of biopsy although overall, the evidence appears to favour continuing such medications. Other RFs are also well described. Patients and practitioners need to be aware of the RFs for the development of rectal bleeding following prostate biopsy, the potential severity of such bleeding and most importantly, the appropriate measures required to manage it. We advocate early consultation with the Colorectal/Gastroenterology and Interventional Radiology services and a progressive, stepwise approach to its management, starting with resuscitation and conservative tamponade measures, moving to endoscopic haemostasis (injection/banding/clipping) +/- radiological (embolization) +/- transanal surgical methods depending on the clinical scenario, available resources, practitioner preference and success of previous attempted corrective measures (see Figure 4).

Major rectal bleeding post TRUS biopsy is a rare occurrence. This is reflected by the fact that most of the cited papers in our study are case reports, compatible with McCulloch's Innovation phase. ${ }^{46}$ To enhance our experience and expertise in managing it, Urologists should be encouraged to report cases of this complication. To facilitate this, centralised registries could be established to capture all such data and a blame-free culture of open reporting needs to be fostered. Future research might also give us a greater understanding and knowledge on the topic. For example, this might include more/larger prospective, randomized controlled trials comparing continued anticoagulation versus no anticoagulation versus withheld anticoagulation, with particular emphasis on the role of NOACs. In addition, studies comparing the efficacy, cost and morbidity of endoscopic versus angiographic management would be of great interest.

MRQ is supported by a Royal College of Surgeons in Ireland (RCSI) Ethicon Foundation Travel Grant 2016 


\section{References}

1. Loeb S, Vellekoop A, Ahmed HU et al. Systematic review of complications of prostate biopsy. Eur Urol 2013;64(6):876-92.

2. European Association of Urology guidelines. https://uroweb.org/guideline/prostatecancer/?type=pocket-guidelines. Accessed January 272017

3. Bul M, van den Bergh RC, Rannikko A et al. Predictors of unfavourable repeat biopsy results in men participating in a prospective active surveillance program. Eur Urol 2012; 61(2):370-7.

4. van den Bergh RC, Vasarainen H, van der Poel HG et al. Short-term outcomes of the prospective multicentre 'Prostate Cancer Research International: Active Surveillance' study. BJU Int_2010; 105(7):956-62.

5. Ahmed HU, Akin O, Coleman JA et al. Transatlantic Consensus Group on active surveillance and focal therapy for prostate cancer. BJU Int 2012; 109(11):1636-47.

6. Lee G, Attar K, Laniado $\mathrm{M}$ et al. Safety and detailed patterns of morbidity of transrectal ultrasound guided needle biopsy of prostate in a urologist-led unit. Int Urol Nephrol 2006;38(2):281-5.

7. Enlund AL, Varenhorst E. Morbidity of ultrasound-guided transrectal core biopsy of the prostate without prophylactic antibiotic therapy. A prospective study in 415 cases. Br J Urol. 1997;79(5):777-80.

8. Brullet E, Guevara MC, Campo R et al. Massive rectal bleeding following transrectal ultrasound-guided prostate biopsy. Endoscopy 2000;32(10):792-5.

9. Al-Otaibi MF, Al-Taweel W, Bin-Saleh S. Disseminated intravascular coagulation following transrectal ultrasound guided prostate biopsy. J Urol 2004;171(1):346.

10. Seymour MA, Oesterling JE. Anterior rectal wall hematoma: complication of transrectal ultrasound-guided biopsy of prostate. Urology 1992;39(2):177-81.

11. Choyke PL, Blei CL, Jaffe MH et al. Prevesical hematoma: a complication of prostatic biopsy. Urol Radiol 1986;8(1):32-4.

12. Van Agt G, Rojare C, Catteau S et al. Severe late rectal bleeding after biopsy of prostate: a case report. Prog Urol. 2010;20(5):389-91.

13. Toren P, Razik R, Trachtenberg J. Catastrophic sepsis and hemorrhage following transrectal ultrasound guided prostate biopsies. Can Urol Assoc J 2010;4(1):E12-4.

14. Asano T, Kobayashi S, Yano $\mathrm{M}$ et al. Continued administration of antithrombotic agents during transperineal prostate biopsy. Int Braz J Urol 2015;41(1):116-23.

15. Yuan LR, Zhang CG, Lu LX et al. Comparison of ultrasound-guided transrectal and transperineal prostate biopsies in clinical application. Zhonghua Nan Ke Xue 2014;20(11):1004-7.

16. Egbers N, Schwenke C, Maxeiner A et al. MRI-guided core needle biopsy of the prostate: acceptance and side effects. Diagn Interv Radiol 2015;21(3):215-21.

17. Syed MI, Chaudhry N, Shaikh A et al. Catheter-directed middle hemorrhoidal artery embolization for life-threatening rectal bleeding. Can J Gastroenterol

2007;21(2):117-23. 
18. Sopart HJ. Hemorrhage requiring transfusion from the rectal venous plexus following transrectal punch biopsy of the prostate. Z Urol Nephrol 1985 Mar;78(3):157-8.

19. Ustundag Y, Yesilli C, Aydemir S et al. A life-threatening hematochesia after transrectal ultrasound-guided prostate needle biopsy in a prostate cancer case presenting with lymphedema. Int Urol Nephrol 2004;36(3):397-400.

20. Baum RD, Slade M. Use of Foley balloon tamponade in transrectal prostate biopsy hemorrhage. Urology 1988 Aug;32(2):181.

21. Davis NF, McGuire BB, Flood HD. Perioperative management of antithrombotic agents in urological surgery. J Clin Urol 2011;4(5):187-96

22. Giannarini G, Mogorovich A, Selli C. Re: transrectal ultrasound-guided biopsy of the prostate: aspirin increases the incidence of minor bleeding complications. Clin Radiol 2008 Dec;63(12):1386-7.

23. Halliwell OT, Yadegafar G, Lane c et al. Transrectal ultrasound-guided biopsy of the prostate: aspirin increases the incidence of minor bleeding complications. Clin Radiol 2008;63(5):557-61.

24. Maan Z, Cutting CW, Patel U et al. Morbidity of transrectal ultrasonography-guided prostate biopsies in patients after the continued use of low-dose aspirin. BJU Int 2003;91(9):798-800.

25. Carmignani L, Picozzi S, Bozzini G et al. Transrectal ultrasound-guided prostate biopsies in patients taking aspirin for cardiovascular disease: A meta-analysis. Transfus Apher Sci 2011;45(3):275-80.

26. Burger W, Chemnitius JM, Kneissl GD et al. Low-dose aspirin for secondary cardiovascular prevention - cardiovascular risks after its perioperative withdrawal versus bleeding risks with its continuation - review and meta-analysis. J Intern Med 2005;257(5):399-414.

27. Culkin DJ, Exaire EJ, Green D et al. Anticoagulation and antiplatelet therapy in urological practice: ICUD/AUA review paper. J Urol 2014;192(4):1026-34

28. Ihezue CU, Smart J, Dewbury KC et al. Biopsy of the prostate guided by transrectal ultrasound: relation between warfarin use and incidence of bleeding complications. Clin Radiol 2005;60(4):459-63.

29. Halliwell OT, Lane C, Dewbury KC. Transrectal ultrasound-guided biopsy of the prostate: should warfarin be stopped before the procedure? Incidence of bleeding in a further 50 patients. Clin Radiol 2006;61(12):1068-9.

30. Meyer JP, Gillatt DA, Lush R et al. Managing the warfarinized urological patient. BJU Int 2003;92(4):351-4.

31. Raheem OA, Casey RG, Galvin DJ et al. Discontinuation of anticoagulant or antiplatelet therapy for transrectal ultrasound-guided prostate biopsies: a single-center experience. Korean J Urol 2012;53(4):234-9.

32. Coscarella M, Viart L, Nguyen P et al. New oral anticoagulants and prostate biopsy: Which usual precaution should we use? Prog Urol 2015;25(9):510-5. 
33. Ramachandran N, MacKinnon A, Allen C et al. Biopsy of the prostate guided by transrectal ultrasound: relation between warfarin use and incidence of bleeding complications. Clin Radiol 2005;60(10):1130.

34. Katsinelos P, Kountouras J, Dimitriadis G et al. Endoclipping treatment of lifethreatening rectal bleeding after prostate biopsy. World J Gastroenterol 2009;15(9):1130-3.

35. Ghani KR, Dundas D, Patel U. Bleeding after transrectal ultrasonography-guided prostate biopsy: a study of 7-day morbidity after a six-, eight- and 12-core biopsy protocol. BJU Int 2004;94(7):1014-20.

36. Yuen JS, Lau WK, Ng LG et al. Clinical, biochemical and pathological features of initial and repeat transrectal ultrasonography prostate biopsy positive patients. Int $J$ Urol 2004;11(4):225-31.

37. Mariappan P, Chong WL, Sundram M et al. Increasing prostate biopsy cores based on volume vs the sextant biopsy: a prospective randomized controlled clinical study on cancer detection rates and morbidity. BJU Int 2004;94(3):307-10.

38. Obek C, Onal B, Ozkan B et al. Is periprostatic local anesthesia for transrectal ultrasound guided prostate biopsy associated with increased infectious or hemorrhagic complications? A prospective randomized trial. J Urol 2002;168(2):558-61.

39. Sieber PR, Rommel FM, Theodoran CG et al. Contemporary prostate biopsy complication rates in community-based urology practice. Urology 2007;70(3):498500.

40. Khan SA, Hu KN, Marder C et al. Hemorrhoidal bleeding following transrectal prostatic biopsy. Etiology and management. Dis Colon Rectum 1982;25(8):817-9.

41. Ozveren B, Turkeri L. Massive rectal bleeding after prostate biopsy controlled by endoclipping in a patient using acetylsalicylic acid. Can Urol Assoc J 2013;7(56):E442-4.

42. Sheikh M, Hussein AY, Kehinde EO et al. Patients' tolerance and early complications of transrectal sonographically guided prostate biopsy: prospective study of 300 patients. J Clin Ultrasound 2005;33(9):452-6.

43. Rietbergen JB, Kruger AE, Kranse R et al. Complications of transrectal ultrasoundguided systematic sextant biopsies of the prostate: evaluation of complication rates and risk factors within a population-based screening program. Urology 1997;49(6):875-80.

44. Petroski RA, Griewe GL, Schenkman NS. Delayed life-threatening hemorrhage after transrectal prostate needle biopsy. Prostate Cancer Prostatic Dis 2003;6(2):190-2.

45. Gonen M, Resim S. Simplified treatment of massive rectal bleeding following prostate needle biopsy. Int J Urol 2004;11(7):570-2.

46. McCulloch P, Altman DG, Campbell WB et al. No surgical innovation without evaluation: the IDEAL recommendations. Lancet 2009;374(9695):1105-12.

47. Dauleh MI, Byrne DJ. Severe bleeding following transrectal Tru-cut prostatic biopsy. Scand J Urol Nephrol 1996;30(2):153-4. 
48. Maatman TJ, Bigham D, Stirling B. Simplified management of post-prostate biopsy rectal bleeding. Urology 2002 Sep;60(3):508.

49. Bekarma HJ, Smart CJ. The use of a urinary catheter and rectal sponge to tamponade severe bleeding following TRUS prostate biopsy. Ann R Coll Surg Engl 2014;96(7):555.

50. Laracy JC, Regalbuto R, Block BL. Massive Rectal Hemorrhage After Prostate Biopsy: A Teachable Moment. JAMA Intern Med 2016;176(8):1064-5.

51. Kilciler M, Erdemir F, Demir E et al. The effect of rectal Foley catheterization on rectal bleeding rates after transrectal ultrasound-guided prostate biopsy. J Vasc Interv Radiol 2008;19(9):1344-6.

52. Kobatake K, Mita K, Kato M. Effect on hemostasis of an absorbable hemostatic gelatin sponge after transrectal prostate needle biopsy. Int Braz J Urol 2015;41(2):337-43.

53. Park BH, Kim JI, Bae SR et al. The effect of ultrasound-guided compression immediately after transrectal ultrasound-guided prostate biopsy on postbiopsy bleeding: a randomized controlled pilot study. Int Urol Nephrol 2017 May 4. [Epub ahead of print]

54. Pacios E, Esteban JM, Breton ML et al. Endoscopic treatment of massive rectal bleeding following transrectal ultrasound-guided prostate biopsy. Scand J Urol Nephrol 2007;41(6):561-2.

55. Braun KP, May M, Helek C et al. Endoscopic therapy of a massive rectal bleeding after prostate biopsy. Int Urol Nephrol 2007;39(4):1125-9.

56. Strate LL, O’Leary MP, Carr-Locke DL et al. Endoscopic treatment of massive rectal bleeding following prostate needle biopsy. Endoscopy 2001;33(11):981-4.

57. Harris MA, Chadwick D, Ward DC. A novel way of controlling rectal bleeding after transrectal ultrasonography-guided prostate biopsies. BJU Int 2004;93(9):1358.

58. Raju GS, Gajula L. Endoclips for GI endoscopy. Gastrointest EndosC 2004;59(2):267-79.

59. Kinney TP, Kozarek RA, Ylvisaker JT et al. Endoscopic evaluation and treatment of rectal hemorrhage after prostate biopsy. Gastrointest Endosc 2001;53(1):117-9.

60. Arroja B, Goncalves C, Silva F et al. Major rectal bleeding following transrectal needle prostate biopsy. Endoscopy 2009;41 Suppl 2:E145.

61. Geraci G, Sciume C, Pisello F et al. Severe rectal bleeding after transrectal US-guided prostate biopsy. Case report. G Chir 2006;27(8-9):321-3.

62. Pisco JM, Martins JM, Correia MG. Internal iliac artery: embolization to control hemorrhage from pelvic neoplasms. Radiology 1989;172(2):337-9.

63. Bilhim T, Pisco J, Rio Tonto $\mathrm{H}$ et al. Unilateral versus bilateral prostatic arterial embolization for lower urinary tract symptoms in patients with prostate enlargement. Cardiovasc Intervent Radiol 2013;36(2):403-11.

64. Ihimoyan A, Maddela R, Kalakada N et al. Severe Life Threatening Rectal Bleed After Prostate Biopsy Requiring Angiographic Therapy: A Case Report.

Gastroenterology Res 2010;3(5):213-215. 
65. Kaneko T, Suzuki T, Matsushita N et al. Transcatheter arterial embolization for bleeding of prostatic artery after prostate biopsy. Nihon Hinyokika Gakkai Zasshi 2003;94(7):693-5.

66. Anjum MI. Pelvic haematoma following trucut biopsy of the prostate. Int Urol Nephrol 1996;28(6):781-5.

67. De Beule T, Carels K, Tejpar S et al. Prostatic biopsy-related rectal bleeding refractory to medical and endoscopic therapy definitively managed by catheterdirected embolotherapy: a case report. J Med Case Rep 2015;9:242.

68. Vallina Vazquez MJ, Gutierrez Julian JM, Vega Gutierrez F et al. Pulsatile perineal hematoma after transrectal prostatic biopsy. Arch Esp Urol 1999;52(9):998-1002. 


\section{Figures and Tables}

Fig. 1. Prisma flow chart outlining accrual of papers for inclusion in our review article.

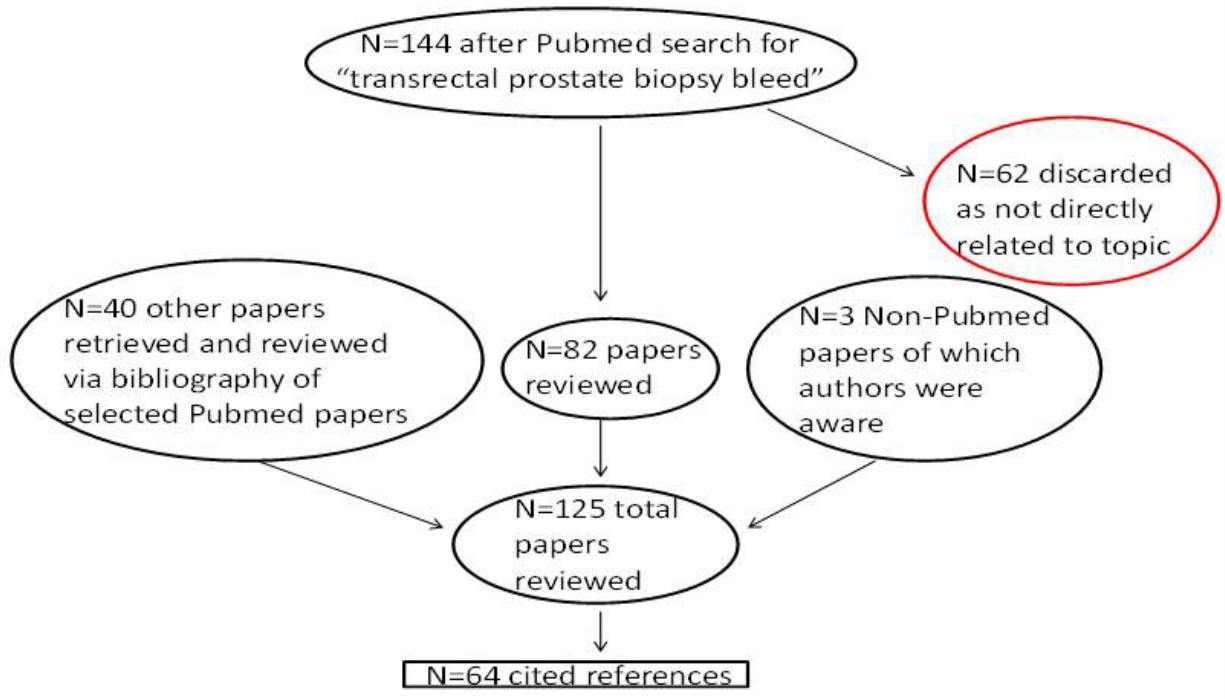

Fig. 2. Images of endoscopic clipping of anterior rectal wall bleeding post-transrectal ultrasound (TRUS) prostate biopsy (reproduced with permission of authors)
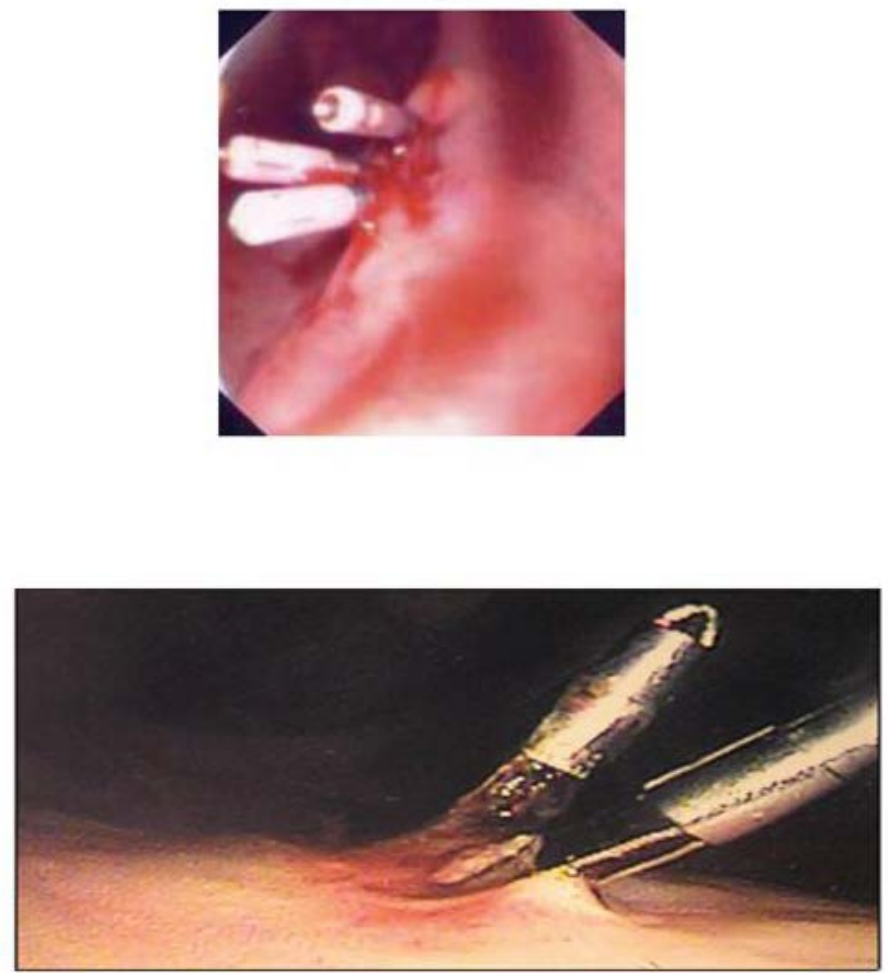
Fig. 3. Control angiography after embolization with microparticles (300-500 $\mu)$ and proximal microcoil (arrows) occlusion of the anastomosis of the left and right inferior prostatic arteries (reproduced with permission of author).

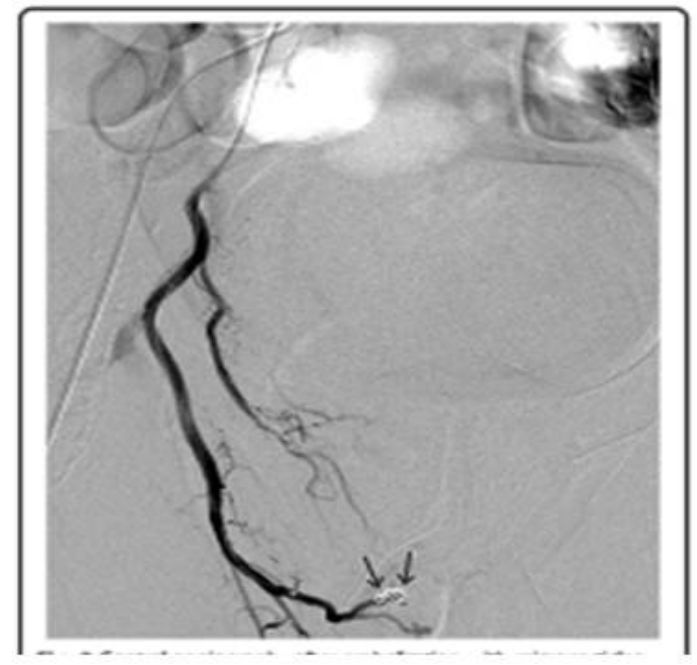

Fig. 4. Proposed hierarchical treatment algorithm for the management of post- transrectal ultrasound (TRUS) biopsy rectal bleeding.

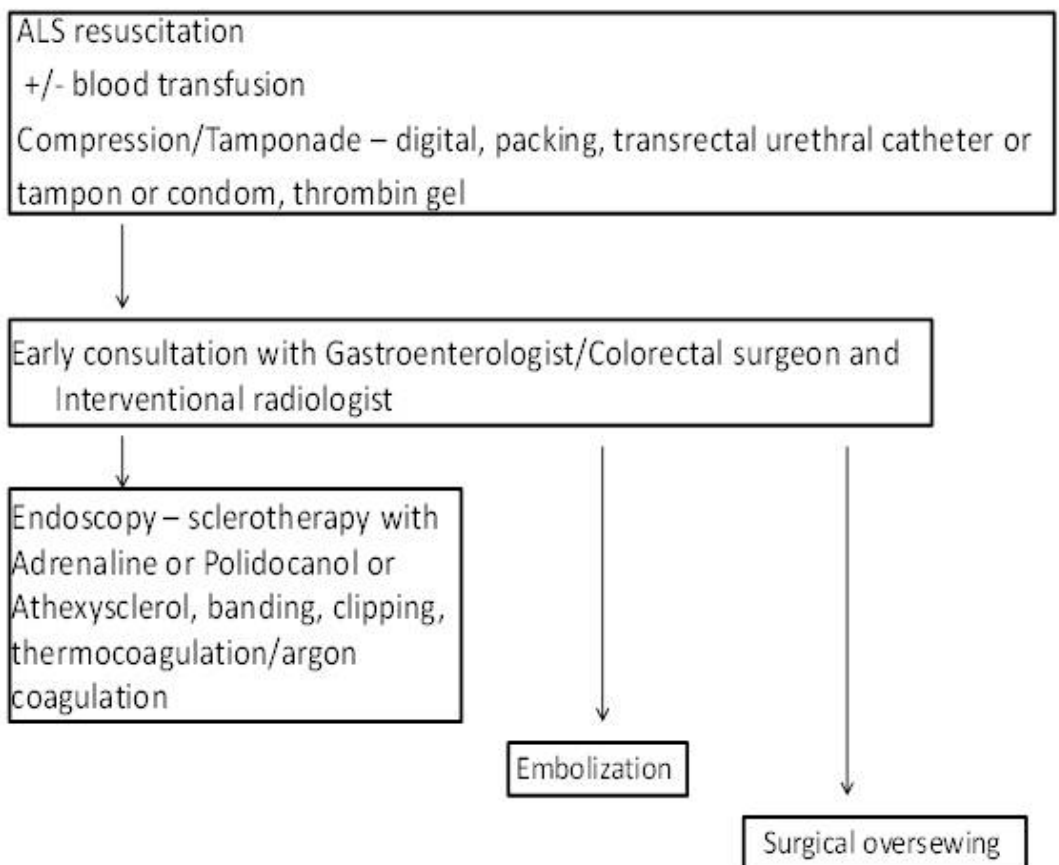




\begin{tabular}{|l|l|}
\hline \multicolumn{2}{|l|}{ Table 1. Levels of evidence as used in our study } \\
\hline Ia & Meta-analysis of randomized trials \\
\hline Ib & $\geq 1$ randomized trial \\
\hline 2a & Well-designed, controlled study without randomization \\
\hline 2b & Well-designed, quasi-experimental study \\
\hline 3 & Non-experimental study (comparative, correlation, case reports) \\
\hline 4 & Expert committee/opinion \\
\hline
\end{tabular}

Table 2. Selected studies of bleeding complications after prostate biopsy

\begin{tabular}{|l|c|c|c|}
\hline First author & Intervention & No. of patients & Rectal bleeding \% \\
\hline Halliwell & ASA vs. nil & 387 vs. 731 & 21 vs. 13 \\
\hline Maan & LDASA vs. nil & 36 vs.141 & 0 vs. 22 \\
\hline Ihezue & Warfarin vs.nil & 49 vs. 902 & 14.3 vs. 13 \\
\hline Raheem & $\begin{array}{c}\text { LDASA, warfarin, } \\
\text { slopidogrel, LMWH } \\
\text { vs. nil }\end{array}$ & 91 vs.98 & 40 vs. 39 \\
\hline Asano ${ }^{*}$ & $\begin{array}{c}\text { ASA, clopidogrel, } \\
\text { warfarin, ticlopidine, } \\
\text { cilostazol vs. nil }\end{array}$ & 84 vs. 84 & 0 vs. 0 \\
\hline
\end{tabular}

${ }^{*}$ Transperineal prostate biopsy. ASA: acetylsalicylic acid; LDASA: low dose acetylsalicylic acid; LMWH: low molecular weight heparin. 